\title{
[O-M-069] MUTATIONS THAT RENDER VON WILLEBRAND FACTOR (VWF) RESISTANT TO ADAMTS13 CLEAVAGE
}

\section{K. Desch, D. Ginsburg. Pediatrics; HHMI, Internal Medicine, Human Genetics, University of Michigan, Ann Arbor, United States}

Introduction: VWF is cleaved specifically by the metalloprotease, ADAMTS13. Loss of VWF cleavage due to ADAMTS13 deficiency results in Thrombotic Thrombocytopenic Purpura (TTP). This study aimed to identify specific amino acid residues within VWF that are critical for substrate recognition and proteolysis by ADAMTS13.

Methods: We introduced random mutations into a previously identified minimal substrate for ADAMTS13, VWF73[1]. Mutants were cloned into a phage display library and screened to identify phage bearing mutant VWF substrates resistant to cleavage. Phage clones from the primary screen were isolated and analyzed in a secondary ELISA screen. Positive clones were subjected to DNA sequence analysis. Selected mutations were introduced into the VWF73 fusion protein and directly analyzed for cleavage by ADAMTS13.

Results: Two independent substrate phage display libraries were screened. 174 individual phage clones were isolated for secondary screening. 103 clones (59\%) were confirmed to be resistant to cleavage on secondary screening. Most of these positive clones encoded amino acid substitutions within the P3 to P2' positions relative to the cleavage site, with a total of 25 unique amino acid substitutions identified. Analysis of selected mutants expressed as fusion proteins confirmed resistance to cleavage by ADAMTS13.

Conclusions: An unbiased genetic approach identified a panel of amino acid substitutions flanking the TYR1605-MET1606 cleavage site that are critical for VWF proteolysis by ADAMTS13. These results suggest that the conformation of VWF immediately surrounding this cleavage site is particularly important for the enzyme-substrate interaction with ADAMTS13. Naturally occurring VWF gene mutations at these positions could result in a TTP-like syndrome, or modify the severity of disease in patients with TTP due to ADAMTS13 deficiency.

References: 1. Kokame, K., et al., VWF73, a region from D1596 to R1668 of von Willebrand factor, provides a minimal substrate for ADAMTS-13. Blood, 2004. 103(2): p. 607-12.

Desch K, Ginsburg D. MUTATIONS THAT RENDER VON WILLEBRAND FACTOR (VWF) RESISTANT TO ADAMTS13 CLEAVAGE. $J$ Thromb Haemost 2007; 5 Supplement 2: O-M-069

Date: Monday, July 9, 2007

Session Info: Oral Communications: TTP / HUS and ADAMTS 13

Room: Room D 